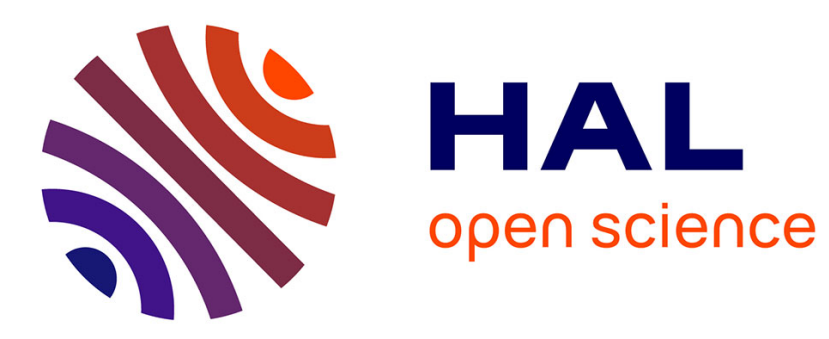

\title{
3D closed-loop swimming at low Reynolds numbers
}

Ali Oulmas, Nicolas Andreff, Stéphane Régnier

\section{To cite this version:}

Ali Oulmas, Nicolas Andreff, Stéphane Régnier. 3D closed-loop swimming at low Reynolds numbers. The International Journal of Robotics Research, 2018, 37 (11), pp.1359-1375. $10.1177 / 0278364918801502$. hal-02093988

\section{HAL Id: hal-02093988 \\ https://hal.sorbonne-universite.fr/hal-02093988}

Submitted on 9 Apr 2019

HAL is a multi-disciplinary open access archive for the deposit and dissemination of scientific research documents, whether they are published or not. The documents may come from teaching and research institutions in France or abroad, or from public or private research centers.
L'archive ouverte pluridisciplinaire HAL, est destinée au dépôt et à la diffusion de documents scientifiques de niveau recherche, publiés ou non, émanant des établissements d'enseignement et de recherche français ou étrangers, des laboratoires publics ou privés. 


\title{
3D closed-loop swimming at low Reynolds numbers
}

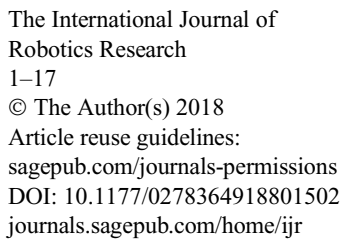

(S)AGE

\author{
Ali Oulmas ${ }^{1,2}$, Nicolas Andreff ${ }^{2}$ and Stéphane Régnier ${ }^{1}$
}

\begin{abstract}
In this paper, the mobility matrix of helical microswimmers is investigated to compute the magnetic torque as a function of the angular velocities of the helical robot to achieve a 3D path following in closed-loop. Thus, the helical swimmer kinematics are expressed in the Serret-Frenet frame considering the weight of the robot and lateral disturbances using the compensation inclination and direction angles, respectively. A new chained formulation is used to design a stable controller. The approach is simple and quite general and can be used for different non-holonomic autonomous systems. The $3 D$ path following is validated by presenting experimental results using a scaled-up helical microswimmer actuated magnetically. Different trajectories were tested: a spatial straight line, a helix trajectory, and an inclined sinusoidal trajectory. Several conditions have been tested experimentally, namely: different velocity profiles, compensation inclination angles, liquid viscosities, control gains and boundary effects, and their impact on the performance of the path following. To illustrate the robustness and accuracy of the visual servo control to disturbances presenting in the environment such as the magnetic field gradient and boundary effects, it is compared with the open-loop control. The results show the robustness of the controller and a submillimetric accuracy during the path following.
\end{abstract}

Keywords

Helical swimmer, magnetic manipulation, 3D path following, visual servoing

\section{Introduction}

At low Reynolds numbers, the locomotion through a fluid is characterized only by viscosity forces, and inertia forces are virtually null. Under these conditions, reciprocal movement is impossible. In nature, microorganisms use a nonreciprocal movement such as the corkscrew-type rotating propulsion, used by the famous Escherichia coli bacteria to swim and navigate. Magnetic helical swimmers reproduce a similar movement using a corkscrew tail and an external rotating magnetic field.

In fact, the miniaturization of robots provides solutions for several applications to access complex and dangerous environments. Magnetic helical swimmers can lead to minimally invasive medicine (Qiu and Nelson, 2015) because magnetic fields do not affect the human body (Siauve, 2003). Injected inside the bloodstream, microswimmers can be used to manipulate cells and transport drugs and microelements (Qiu et al., 2015). Medina-Sánchez et al. (2015) managed to capture, transport, and release an immotile live sperm cell for fertilization assistance. In lab-on-chips, they can be used to sort and transport microobjects, and even to assemble them (Chaillet and Régnier, 2013). Tottori et al. (2012) conceived a microswimmer with a micro-tube capable of pick-and-place tasks.
Thus, to perform such tasks, the motion of the helical swimmers should be robust and accurate to overcome the environmental disturbances such as friction, viscous drag, boundary effects, and strong flow in blood vessels and achieve a certain repeatability to perform real tasks.

Several methods for microswimmer motion control are used by researchers. For instance, Mahoney et al. (2011) had a helical swimmer make a U-turn by estimating the magnetic field rotation direction. Similarly, Ghosh and Fischer (2009) used more complex curves, tracing the letters "R", “@”, or "H $\mathcal{H}$ ". Tottori et al. (2012) used a helical swimmer capable of navigating in space for cargo transport tasks. However, because these methods are open-loop, they are exposed to modeling errors and drifts.

Controlling the magnetic helical swimmers in closedloop will make them less sensitive to modeling errors and

\footnotetext{
${ }^{1}$ Sorbonne Université, CNRS, UMR 7222, ISIR, Paris, France

${ }^{2}$ FEMTO-ST Institute, University Bourgogne Franche-Comté/CNRS, Besançon, France
}

Corresponding author:

Ali Oulmas, Intelligent Systems and Robotics Institute, ISIR, Sorbonne Université, CNRS, 75005 Paris, France.

Email: ali.oulmas@isir.upmc.fr 
environmental disturbances such as boundary effects and thermal noises. Xu et al. (2015) proposed a planar pathfollowing control to improve the accuracy and robustness of these helical swimmers. The algorithm was validated using a scaled-up helical microswimmer by following a straight line in the horizontal plane. However, the altitude was kept (almost) constant by an open-loop control, thus limiting the motion to planar horizontal curves. The kinematic model used by $\mathrm{Xu}$ et al. (2015) was developed by Samson (1995) for planar path following of wheeled robots and is not adapted to follow a path in 3D space. In Oulmas et al. (2016b), we extended the approach to a general pathfollowing algorithm for non-holonomic systems. The robot was modeled in a local frame using the Serret-Frenet frame instead of the global frame, taking into account the weight of the non-holonomic system and lateral disturbances. The resulting system was nonlinear, a new chained system was used to design the controller with exact linearization. As the helical robot behaves as a non-holonomic system, the approach was tested on experimentation using a scaled-up helical microswimmer in Oulmas et al. (2016a) by following different $3 \mathrm{D}$ geometrical paths. In this paper, for the first contribution, the 3D path-following controller is developed in detail for the helical swimmer case and tested on different curve shapes under different conditions such as the boundary effects and the change of viscosity that typically dominate the motion of these swimmers.

The second contribution is a coherent study of the 3D path-following problem for non-holonomic helical swimmers starting with dynamic equations at low Reynolds numbers $(\operatorname{Re} \ll 1)$ to express the magnetic torque necessary to drive the helical swimmer to follow a 3D geometric path as a function of the steering angular velocity of the robot, which is computed thanks to the kinematic controller.

This approach is validated using a scaled-up helical microswimmer actuated magnetically by following different trajectories with different curvatures, inclinations, and torsions in space. Several conditions are also tested: different velocity profiles (step input, rectangular signal input, and a continuous arbitrary signal input); different boundary-path distances; different fluid viscosities using glycerol; different compensation inclination angles; and different control gains. Furthermore, to illustrate the robustness and accuracy of the visual servo control to different kinds of disturbances such as the weight of the robot and modeling errors, it is compared with the open-loop control.

In the remainder of this paper, Section 2 presents the electromagnetic manipulation system used to steer the helical swimmer wirelessly and the different efforts applied on the helical robot. Afterwards, Section 3 describes the state of the art of 3D path following for non-holonomic systems and then gives the 3D error kinematic model of the helical swimmer using the Serret-Frenet frame with compensation direction and inclination angles. Section 4 shows the conversion of the kinematic model into the chained form, the control law design for 3D path following, and the magnetic

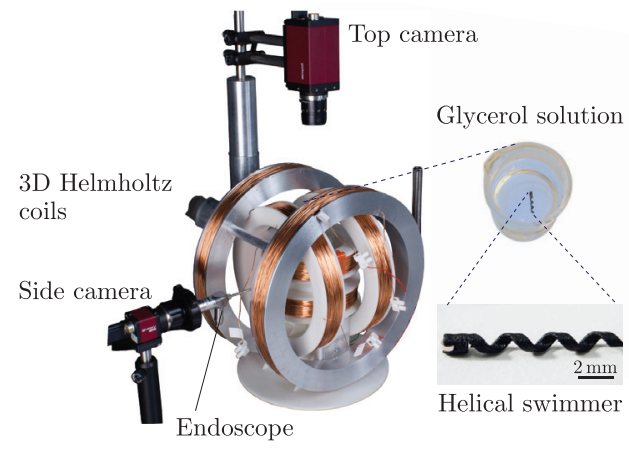

Fig. 1. Magnetic manipulation system.

controller for the self-rotation and steering of the helical robot. Section 5 shows the results obtained by applying the $3 \mathrm{D}$ visual servo control on the prototype.

\section{Magnetic manipulation system}

In this work, vision-based detection and tracking methods are used to locate and follow the helical swimmer (ViSP; Marchand et al., 2005). The robot is manufactured using the 3D printer with the VisiJet M3-black material. Then, a cylindrical permanent magnet is attached to the head of the helix in a manner to have the magnetization perpendicular to the principal axis of the helical robot.

\subsection{Magnetic torque and force}

The helical swimmer is wirelessly actuated in space using external rotating magnetic fields. The actuated system is composed of three pairs of Helmholtz coils as shown in Figure 1. Each coil pair generates a uniform magnetic field along an axis. The magnetic torque $\mathbf{T}_{m}$ applied on the helical swimmer with a magnetic moment $\mathbf{M}$ in an external magnetic field $\mathbf{B}$, is given by

$$
\mathbf{T}_{m}=\mathbf{M} \times \mathbf{B}
$$

The magnetic torque tends to align the magnetic moment with the applied magnetic field (Coey, 2010). Therefore, with a rotating magnetic field and a helical tail, the helical swimmer can advance by converting its self-rotation into linear motion. However, the experimental magnetic field $\mathbf{B}$ is not perfectly uniform. Therefore, a magnetic force $\mathbf{F}_{m}$ is generated and is given as follows:

$$
\mathbf{F}_{m}=\nabla(\mathbf{M} \cdot \mathbf{B})
$$

where $\nabla$ is the gradient operator (Coey, 2010). The presence of $\mathbf{F}_{m}$ results in a translation of the helical swimmer in the same direction as $\mathbf{F}_{m}$. The imperfections of $\mathbf{B}$ are hard to model, generally small and hard to exploit. Therefore, we consider the force $\mathbf{F}_{m}$ and the resulting translation as a disturbance that has to be rejected by a closed-loop control. 


\subsection{Dynamic equations}

When the helical robot is swimming at low Reynolds numbers, the inertia is being neglected, the drag force and torque due to the translational and rotational motions of the body balance the non-fluidic applied force and torque $\mathbf{F}$ and $\mathbf{T}$, respectively, as follows:

$$
\begin{aligned}
& \mathbf{F}+\mathbf{F}_{\text {drag }}=0 \\
& \mathbf{T}+\mathbf{T}_{\text {drag }}=0
\end{aligned}
$$

Developing (1) and (2) yields an expression of the propulsion velocity $\mathbf{v}_{p}$ and the angular velocity $\boldsymbol{\Omega}$ of the helical swimmer as a function of $\mathbf{F}$ and $\mathbf{T}$, which can be represented by the following symmetric mobility matrix as defined in Fu et al. (2015):

$$
\left[\begin{array}{l}
\mathbf{v}_{p} \\
\boldsymbol{\Omega}
\end{array}\right]=\left[\begin{array}{cc}
\mathbf{A} & \mathbf{D} \\
\mathbf{D}^{\mathrm{T}} & \mathbf{C}
\end{array}\right]\left[\begin{array}{l}
\mathbf{F} \\
\mathbf{T}
\end{array}\right]
$$

where $\mathbf{A}, \mathbf{D}$ and $\mathbf{C}$ are $3 \times 3$ submatrices forming the mobility matrix. These elements depend on the shape and length of the helical swimmer and also on the viscosity of the liquid. The inverse of the mobility matrix gives the propulsion matrix as in Mahoney et al. (2011).

From (3), the angular velocity of the helical swimmer can be given as

$$
\boldsymbol{\Omega}=\mathbf{D}^{\mathrm{T}} \mathbf{F}+\mathbf{C T}
$$

The non-fluidic force $\mathbf{F}$ is given by the sum of gravity and magnetic forces (i.e. $\mathbf{F}=\mathbf{F}_{g}+\mathbf{F}_{m}$, where $\mathbf{F}_{g}$ is the gravity force and $\mathbf{F}_{m}$ is the magnetic force).

In this section, the dynamic of the helical swimmer is represented at low scale, which means that the fluid is characterized by low Reynolds numbers and the swimmer gravity force is null $\left(\mathbf{F}_{g}=0\right)$. The latter assumption started from the fact that the helical swimmer behaves similarly to bacteria, which are approximately neutrally buoyant. However, as the weight of the scaled-up helical microswimmer used in this paper is not null, the gravity force is considered as a disturbance that has to be corrected using the kinematic controller.

In addition, because the magnetic field is uniform, the magnetic force is null $\left(\mathbf{F}_{m}=0\right)$. Thus, the non-fluidic applied force $\mathbf{F}$ can be neglected and the helical swimmer angular velocity in (4) can be simplified as follows:

$$
\boldsymbol{\Omega}=\mathbf{C T}
$$

where $\mathbf{C}$ has the following form:

$$
\mathbf{C}=\left[\begin{array}{ccc}
c_{11} & 0 & c_{13} \\
0 & c_{22} & 0 \\
c_{31} & 0 & c_{33}
\end{array}\right]
$$

This submatrix is not diagonal because of the helical geometry of the swimmer. More details can be found in $\mathrm{Fu}$ et al. (2015) and Mahoney et al. (2011).
As $\mathbf{C}$ is invertible, the magnetic torque $\mathbf{T}$ can be expressed as follows:

$$
\mathbf{T}=\mathbf{C}^{-1} \boldsymbol{\Omega}
$$

Thus, the purpose of the kinematic controller developed in the next section is to compute the angular velocity $\boldsymbol{\Omega}$ necessary to generate a magnetic torque $\mathbf{T}$ to control the helical robot to follow a 3D geometric path.

\section{Kinematic model in the Serret-Frenet frame of a helical swimmer in space}

\subsection{State of the art}

The helical swimmer advances forward (or backward) in the same direction as its principal axis. Constraints on the swimmer geometry prevent it from moving directly sideways. The helical swimmer approximates a non-holonomic system. The lateral displacements generated by boundary effects and thermal noises are considered as disturbances, and should be corrected using the closed-loop control. Considering the helical swimmer as a non-holonomic system allows to use the control laws of non-holonomic autonomous robots such as underwater vehicles and aerial aircrafts.

3D path following has been studied extensively in the literature for non-holonomic autonomous robots in closedloop. Several control laws have been presented: sliding mode techniques are used for path following of an autonomous underwater vehicle (AUV) in Healey and Lienard (1993). These techniques have been shown to be simple but present some drawbacks such as the chattering that can damage the actuators; backstepping methods using Lyapunov theory are also used for path following of an AUV in Encarnacao and Pascoal (2000).

Samson (1995) developed a planar path-following controller of wheeled robots. The method consists of linearizing the kinematic model using the chained form and then applying a linear control law. Wadoo et al. (2012) have proposed an approach to extend this method for trajectory tracking of underwater vehicles. However, the resulting system is linear time-varying (LTV) and not fully decoupled.

Our proposition is to extend the method in Samson (1995) into a general form for 3D path following of helical swimmers. In fact, during disturbances, decoupling path following and the velocity profile presents better performances with regards to accuracy because the environmental conditions and actuation constraints impose a saturation in the velocity amplitude of the robot. In addition, most often, the task to be realized by the helical swimmer will be a geometric path to follow, without any specification in velocity profile.

\subsection{Notation}

The notation for the 3D path following of the helical swimmer is summarized in Table 1. 
Table 1. Notations for 3D path following.

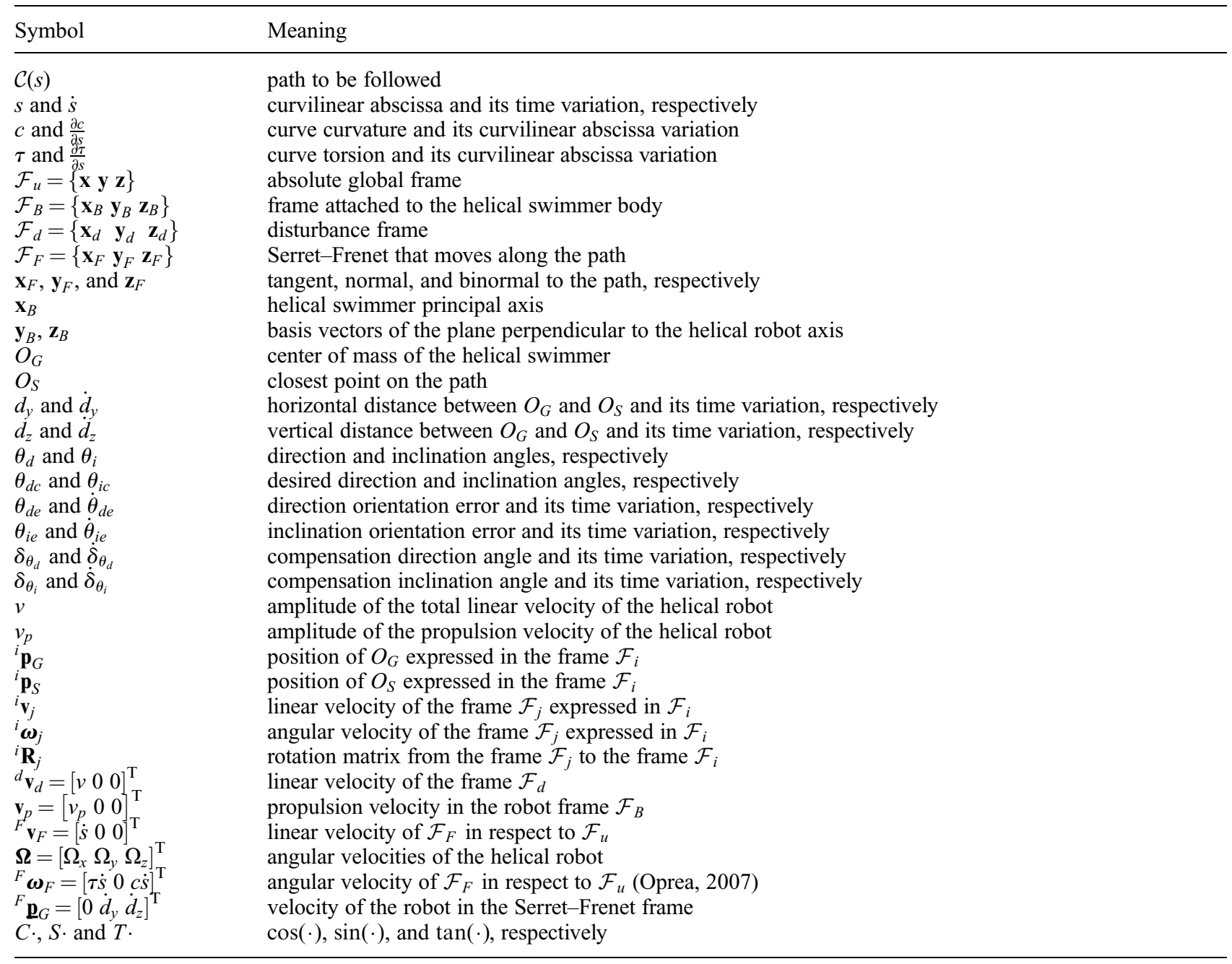

\subsection{Kinematic equations}

To control the helical swimmer, a mathematical model describing its behavior is necessary. Therefore, an error kinematic model with the Serret-Frenet frame associated to the reference path is used to express the desired motion in terms of the path parameters. The non-holonomic constraints are also considered. Figure 3 represents the block diagram of the closed-loop control for 3D path following of the magnetic helical swimmer.

The kinematics of the helical swimmer with nonholonomic constraints are developed using an absolute global frame $\mathcal{F}_{u}$ with origin $O$ and a moving body frame $\mathcal{F}_{B}$ attached to the body of the helical swimmer located at $O_{G}$ the center of mass of the robot. The orientation of the robot is characterized by the inclination angle $\theta_{i}$ and the direction angle $\theta_{d}$. The former is the angle of the helical robot axis with respect to the horizontal plane $\mathbf{x} O \mathbf{y}$ (pitch angle) and the latter is the angle of the helical robot in the horizontal plane (yaw angle), as illustrated in Figure 2.

During disturbances, the swimmer total linear velocity $\mathbf{v}$ is not aligned with the swimmer principal axis $\mathbf{x}_{B}$. Thus, the disturbance frame $\mathcal{F}_{d}$ is defined as the frame where the $\mathbf{x}_{d}$ axis is along the swimmer total linear velocity $\mathbf{v}$. In the case of aircraft, this frame is called "the wind frame" referencing to the disturbances related to the wind (Kaminer et al., 2006; Oliveira et al., 2013). The compensation direction angle $\delta_{\theta_{d}}$ is used to compensate for lateral

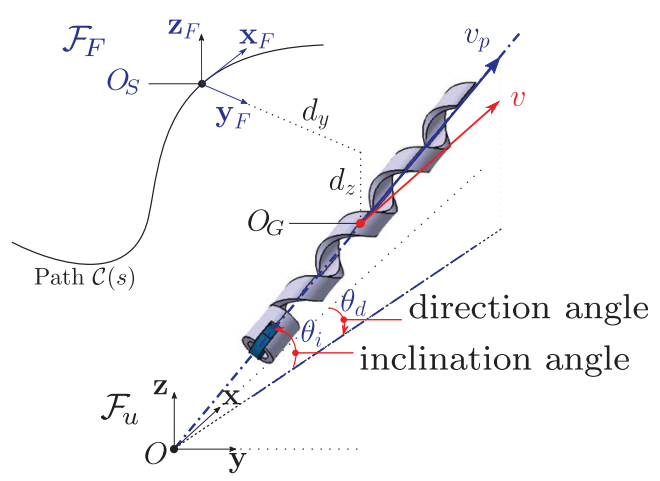

Fig. 2. 3D path following of a helical swimmer. 


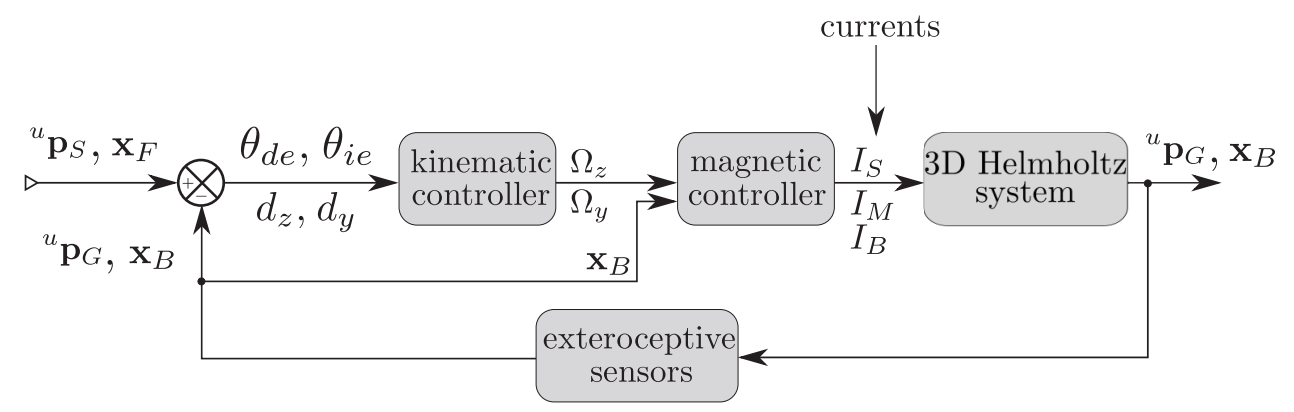

Fig. 3. Block diagram for the $3 \mathrm{D}$ path following of a helical swimmer.

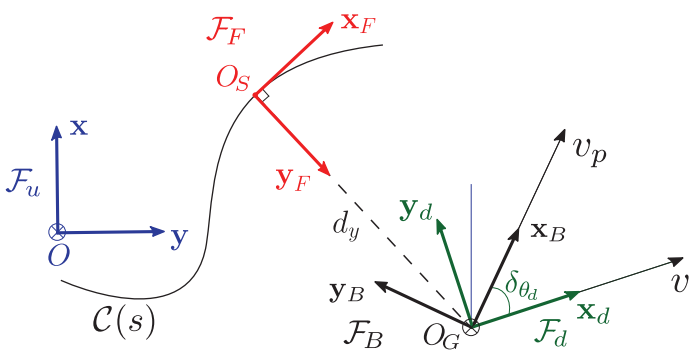

(a)

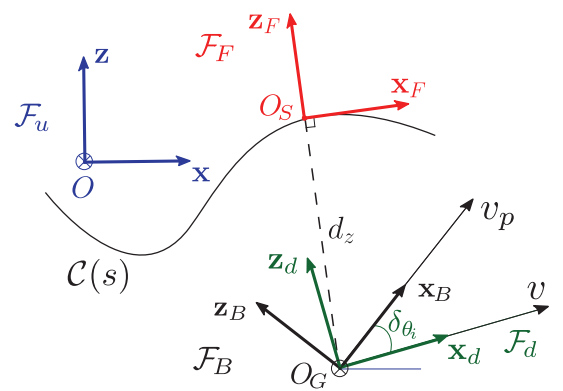

(b)

Fig. 4. Representation of different frames used in 3D path following of the helical robot.

disturbances. It is defined as the angle between $\mathbf{x}_{B}$ and $\mathbf{x}_{d}$ in the horizontal plane as illustrated in Figure 4a. The compensation inclination angle $\delta_{\theta_{i}}$ is used to compensate for disturbances in the vertical plane (such as the robot weight in our experimental set-up with a scaled-up helical microswimmer). It is defined as the angle between $\mathbf{x}_{B}$ and $\mathbf{x}_{d}$ as illustrated in Figure $4 \mathrm{~b}$.

The rotation matrix from $\mathcal{F}_{d}$ to $\mathcal{F}_{B}$ corresponds to the sequence of rotation $\left(-\delta_{\theta_{d}}, \delta_{\theta_{i}}, 0\right)$ and is given as in Etkin (2012) by

$$
{ }^{B} \mathbf{R}_{d}=\left[\begin{array}{ccc}
C \delta_{\theta_{i}} C \delta_{\theta_{d}} & -C \delta_{\theta_{i}} S \delta_{\theta_{d}} & -S \delta_{\theta_{i}} \\
S \delta_{\theta_{d}} & C \delta_{\theta_{d}} & 0 \\
S \delta_{\theta_{i}} C \delta_{\theta_{d}} & -S \delta_{\theta_{i}} S \delta_{\theta_{d}} & C \delta_{\theta_{i}}
\end{array}\right]
$$

As indicated in Table 1, the symbols $C$. and $S$. denote the trigonometric functions $\cos (\cdot)$ and $\sin (\cdot)$, respectively.

The total linear speed $v$ can be computed using the propulsion speed $v_{p}$ along the helical swimmer axis $\mathbf{x}_{B}$, as follows:

$$
v=\frac{1}{C \delta_{\theta_{i}} C \delta_{\theta_{d}}} v_{p}
$$

where $v_{p}$ can be computed thanks to the propulsion matrix in (3).

The path to be followed is denoted by $\mathcal{C}(s)$, which is described with the curvilinear coordinate $s$, the curvature and torsion of the path being $c$ and $\tau$, respectively.
Here $\mathcal{F}_{F}$ is the Serret-Frenet frame that moves along the path $\mathcal{C}(s)$ and $\mathbf{x}_{F}$ and $\mathbf{y}_{F}$ are tangent and normal to the path, respectively, whereas $\mathbf{z}_{F}$ represents the binormal to the path and is given by the cross product of $\mathbf{x}_{F}$ and $\mathbf{y}_{F}$. The SerretFrenet frame $\mathcal{F}_{F}$ is characterized by the reference direction angle $\theta_{d c}$ and the reference inclination angle $\theta_{i c}$. The different frames and orientations used for $3 \mathrm{D}$ path following of helical swimmer are depicted in Figure 4.

The aim of the path-following problem is to minimize the distance and orientation errors between the robot and the reference path. For that purpose, the following state vector is defined:

$$
\mathbf{q}=\left[\begin{array}{c}
s \\
d_{y} \\
\theta_{d e} \\
d_{z} \\
\theta_{i e}
\end{array}\right]=\left[\begin{array}{c}
s \\
d_{y} \\
\theta_{d}-\delta_{\theta_{d}}-\theta_{d c} \\
d_{z} \\
\theta_{i}-\delta_{\theta_{i}}-\theta_{i c}
\end{array}\right]
$$

where $d_{y}$ and $d_{z}$ represent the vertical and horizontal distances between the robot center of mass $O_{G}$ and the point $O_{S}$ closest to the path, respectively, whereas $\theta_{d e}$ and $\theta_{i e}$ represent the direction and inclination angle errors, respectively.

The position ${ }^{u} \mathbf{p}_{G}$ of the robot in the global frame $\mathcal{F}_{u}$ can be expressed as in Figure 2:

$$
{ }^{u} \mathbf{p}_{G}={ }^{u} \mathbf{p}_{S}+{ }^{u} \mathbf{R}_{F}{ }^{F} \mathbf{p}_{G}
$$


where ${ }^{u} \mathbf{R}_{F}$ is the rotation matrix from $\mathcal{F}_{F}$ to $\mathcal{F}_{u}$ and ${ }^{u} \mathbf{p}_{S}$ is the closest point on the path expressed in the global frame $\mathcal{F}_{u}$ whereas ${ }^{F} \mathbf{p}_{G}$ is the position of the robot in the SerretFrenet frame.

Differentiating (6) with respect to the time and expressing it in the Serret-Frenet frame gives

$$
{ }^{F} \mathbf{R}_{d}{ }^{d} \mathbf{v}_{d}={ }^{F} \mathbf{v}_{F}+{ }^{F} \dot{\mathbf{p}}_{G}+{ }^{F} \boldsymbol{\omega}_{F} \times{ }^{F} \mathbf{p}_{G}
$$

where ${ }^{F} \mathbf{R}_{d}$ is the rotation matrix from $\mathcal{F}_{d}$ to $\mathcal{F}_{F}$ using Euler angles and is given by

$$
{ }^{F} \mathbf{R}_{d}=\left[\begin{array}{ccc}
C \theta_{d e} C \theta_{i e} & -S \theta_{d e} & C \theta_{d e} S \theta_{i e} \\
S \theta_{d e} C \theta_{i e} & C \theta_{d e} & S \theta_{d e} S \theta_{i e} \\
-S \theta_{i e} & 0 & C \theta_{i e}
\end{array}\right]
$$

The angle rates $\dot{\theta}_{i e}$ and $\dot{\theta}_{d e}$ are computed from the relative angular velocity between the Serret-Frenet frame $\mathcal{F}_{F}$ and the frame $\mathcal{F}_{d}$ as follows:

$$
{ }^{F} \dot{\mathbf{R}}_{d}={ }^{F} \mathbf{R}_{d} \mathbf{S k}\left({ }^{d} \boldsymbol{\omega}_{d, F}^{r}\right)
$$

where $\mathbf{S k}(\cdot)$ is a skew-symmetric matrix and ${ }^{d} \boldsymbol{\omega}_{d, F}^{r}$ is the relative angular velocity that is given as shown in Encarnacao and Pascoal (2000) by

$$
{ }^{d} \boldsymbol{\omega}_{d, F}^{r}={ }^{d} \boldsymbol{\omega}_{B}+{ }^{d} \boldsymbol{\omega}_{d, B}^{r}-{ }^{d} \boldsymbol{\omega}_{F}
$$

More details on the development of Equations (6) and (8) are given in Appendix B.

Solving (7) for $\dot{s}, \dot{d}_{y}$ and $\dot{d}_{z}$ and (8) for $\dot{\theta}_{i e}$ and $\dot{\theta}_{d e}$ gives the following $3 \mathrm{D}$ error kinematic model of helical swimmers:

$$
\begin{gathered}
\dot{s}=\frac{v C \theta_{d e} C \theta_{i e}}{1-c d_{y}} \\
\dot{d}_{y}=v S \theta_{d e} C \theta_{i e}+\tau d_{z} \dot{s} \\
\dot{d}_{z}=-v S \theta_{i e}-\tau d_{y} \dot{s} \\
\dot{\theta}_{i e}=\Omega_{y} C \delta_{\theta_{d}}-\Omega_{z} S \delta_{\theta_{d}} S \delta_{\theta_{i}}-\dot{\delta}_{\theta_{i}} C \delta_{\theta_{d}}+\tau \dot{s} S \theta_{d e} \\
\dot{\theta}_{d e}=\Omega_{z} \frac{C \delta_{\theta_{i}}}{C \theta_{i e}}+\frac{\dot{\delta}_{\theta_{d}}}{C \theta_{i e}}-\tau \dot{s} T \theta_{i e} C \theta_{d e}-c \dot{s}
\end{gathered}
$$

Note that these equations are not defined at $d_{y}=\frac{1}{c}$ and $\theta_{i e}$ must differ from $\frac{\pi}{2}[\pi]$.

The projection of the model (9) in the horizontal plane by setting the variables $d_{z}, \tau, \delta_{\theta_{i}}, \theta_{i e}$, and $\Omega_{y}$ to zero gives the kinematic model used by Xu et al. (2015), Samson (1995), and Morin and Samson (2008) for planar path following of mobile robots:

$$
\begin{aligned}
& \dot{s}=\frac{v C \theta_{d e}}{1-c d_{y}} \\
& \dot{d}_{y}=v S \theta_{d e} \\
& \dot{\theta}_{d e}=\Omega_{z}+\dot{\delta}_{\theta_{d}}-c \dot{s}
\end{aligned}
$$

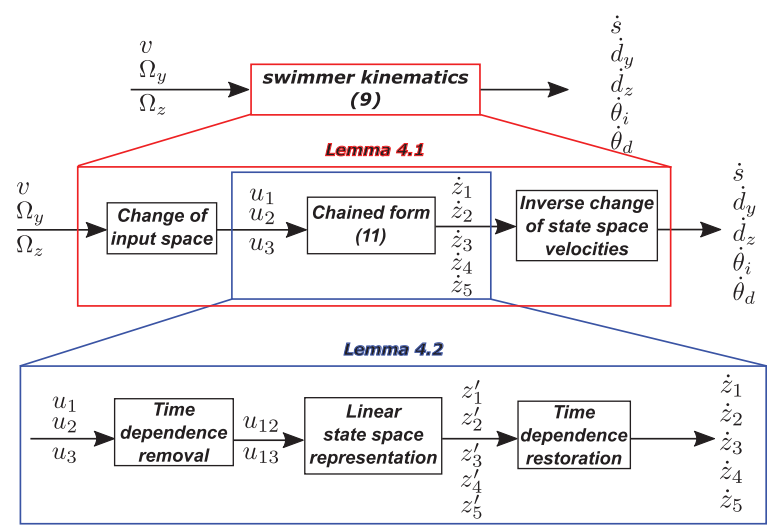

Fig. 5. The linearization of the helical swimmer kinematic model using Lemmas 4.1 and 4.2 .

\section{Chained form and control law}

\subsection{Chained form}

The scaled-up helical microswimmer weight and lateral disturbances are modeled using the disturbance frame $\mathcal{F}_{d}$. Thus, during disturbances, the 3D path following consists of aligning the swimmer total linear velocity $\mathbf{v}$ with the reference path tangent $\mathbf{x}_{F}$ instead of aligning the propulsion velocity $\mathbf{v}_{p}$ with the reference path tangent $\mathbf{x}_{F}$, i.e. the distances $d_{y}$ and $d_{z}$ and the orientations $\theta_{i e}$ and $\theta_{d e}$ must be servoed to zero. For that purpose, a stable control law must be synthesized:

$$
\left(v, \Omega_{y}, \Omega_{z}\right)=f\left(d_{y}, d_{z}, \theta_{i e}, \theta_{d e}\right)
$$

The angular velocity $\Omega_{x}$ along the helical robot axis is not considered because the error in roll does not perturb the path following (Encarnacao and Pascoal, 2000). In addition, the helical robot is propelled externally by a rotating magnetic field along its principal axis.

Samson (1995) proposed a method to linearize the kinematic models of wheeled robots (unicycle, car-like, with trailer) using the chained form with two inputs and three states to design a simple and efficient controller.

In this paper, we propose to convert the kinematic model formed by (9) into a linear model using a new chained formulation with three inputs and five states building on work carried out by Bushnell et al. (1995) and Walsh and Bushnell (1995) on the linearization of multiinputs non-holonomic autonomous systems using the chained form and also on the work of Morin and Samson (2008).

Considering the dependence of the set of equations (9) to $\dot{s}$ and partial decoupling between the planar and vertical planes, those equations can be transformed by appropriate changes of coordinates in a so-called chained form. Figure 5 represents the different steps to linearize the helical swimmer kinematic model using the chained form system.

Lemma 4.1. The change of state space 


$$
\begin{aligned}
& z_{1}=s \\
& z_{2}=d_{y} \\
& z_{3}=\tau d_{z}+\left(1-c d_{y}\right) T \theta_{d e} \\
& z_{4}=d_{z} \\
& z_{5}=-\tau d_{y}+\left(c d_{y}-1\right) T \theta_{i e}\left(C \theta_{d e}\right)^{-1}
\end{aligned}
$$

and control inputs

$$
\begin{aligned}
& u_{1}=\frac{v C \theta_{d e} C \theta_{i e}}{1-c d_{y}} \\
& u_{2}=\gamma_{21} \Omega_{z}+\gamma_{22} \\
& u_{3}=\gamma_{31} \Omega_{y}+\gamma_{32} \Omega_{z}+\gamma_{33}
\end{aligned}
$$

converts the kinematic model (9) of the helical swimmer into the following chained form system with three inputs and five states:

$$
\begin{gathered}
\dot{z}_{1}=u_{1} \\
\dot{z}_{2}=z_{3} u_{1} \\
\dot{z}_{3}=u_{2} \\
\dot{z}_{4}=z_{5} u_{1} \\
\dot{z}_{5}=u_{3}
\end{gathered}
$$

where $\boldsymbol{z}_{l}=\left(z_{1}, z_{2}, z_{3}, z_{4}, z_{5}\right)^{\mathrm{T}}$ is the state vector and $\boldsymbol{u}_{l}=\left(u_{1}, u_{2}, u_{3}\right)^{\mathrm{T}}$ is the input vector. These transformations are not defined if the orientation errors $\theta_{i e}$ and $\theta_{d e}$ are equal to $\frac{\pi}{2}[\pi]$ and the horizontal distance error $d_{y}$ to $\frac{1}{c}$.

The interest of the chained form is that the control input $u_{2}$ yields the convergence of $z_{2}$ and $\dot{z}_{2}$ to zero, then from (11b), this implies the convergence of $z_{3}$ to zero also in the case where $u_{1}$ is non-zero. The same method can be applied using the control input $u_{3}$ to show the convergence of $z_{4}$ and $z_{5}$ to zero. For that, $u_{1}$ is chosen as the velocity along the reference path which is always non-zero (i.e. $\left.u_{1}=\operatorname{arbitrary} \neq 0\right), z_{2}$ and $z_{4}$ as the distance errors to the reference trajectory whereas $z_{3}$ and $z_{5}$ represent the orientation errors with anticipation of the path variation (torsion, curvature).

Proof. The transformations of the kinematic model (9) into the canonical chained form (11) start by choosing the first variable state as

$$
z_{1}=s
$$

Thus, from (9a):

$$
u_{1}=\dot{z}_{1}=\dot{s}=\frac{v C \theta_{d e} C \theta_{i e}}{1-c d_{y}}
$$

The input $u_{1}$ is a function of the total linear velocity $v$. Then, the second variable state is chosen as the horizontal distance:

$$
z_{2}=d_{y}
$$

Thus, from (9b):

$$
\dot{z}_{2}=\dot{d}_{y}=v S \theta_{d e} C \theta_{i e}+\tau d_{z} \dot{S}
$$

Then, from (11b), (12), and (14), $z_{3}$ is obtained as

$$
z_{3}=\left(1-c d_{y}\right) T \theta_{d e}+\tau d_{z}
$$

Therefore, from (11c) we have

$$
\begin{gathered}
u_{2}=\dot{z}_{3} \\
=\tau \dot{d}_{z}+d_{z} \dot{s} \frac{\partial \tau}{\partial s}+\left(1-c d_{y}\right) \dot{\theta}_{d e}\left(C \theta_{d e}\right)^{-2} \\
-\left(c \dot{d}_{y}+d_{y} \dot{s} \frac{\partial c}{\partial s}\right) T \theta_{d e}
\end{gathered}
$$

where $\theta_{d e}$ and $\theta_{i e}$. must differ from $\frac{\pi}{2}[\pi]$ and $d_{y}$ from $\frac{1}{c}$.

Replacing $\dot{s}, \dot{d}_{y}, \dot{d}_{z}$, and $\dot{\theta}_{d e}$ by their values in (9), the input $u_{2}$ is obtained as a function of the steering angular velocity $\Omega_{z}$ :

$$
u_{2}=\gamma_{21} \Omega_{z}+\gamma_{22}
$$

where $\gamma_{21}$ and $\gamma_{22}$ are the following scalar variables:

$$
\begin{aligned}
& \gamma_{21}=v \dot{s}^{-1}\left(C \theta_{d e}\right)^{-1} C \delta_{\theta_{i}} \\
& \gamma_{22}=v \dot{s}^{-1}\left(C \theta_{d e}\right)^{-1} \dot{\delta}_{\theta_{d}}- \\
& \dot{s}\left(2 v \dot{s}^{-1} \tau S \theta_{i e}+\tau^{2} d_{y}-d_{z} \frac{\partial \tau}{\partial s}+c\left(c d_{y}-1\right)\left(1-2\left(C \theta_{d e}\right)^{-2}\right)\right. \\
& \left.+\left(c \tau d_{z}+d_{y} \frac{\partial c}{\partial s}\right) T \theta_{d e}\right)
\end{aligned}
$$

Note that $\gamma_{22}$ depends on the total linear velocity $v$ of the robot and the time derivative of the compensation direction angle $\dot{\delta}_{\theta_{d}}$.

In the same way, the fourth state variable is chosen as the vertical distance:

$$
z_{4}=d_{z}
$$

Thus, from (9d):

$$
\dot{z}_{4}=\dot{d}_{z}=-v S \theta_{i e}-\tau d_{y} \dot{s}
$$

From (11d), (12), and (18), $z_{5}$ comes as

$$
z_{5}=\left(c d_{y}-1\right) T \theta_{i e} C\left(\theta_{d e}\right)^{-1}-\tau d_{y}
$$

Finally, from (11e) and (19):

$$
\begin{aligned}
& u_{3}=\dot{z}_{5} \\
& =-\tau \dot{d}_{y}-d_{y} \dot{s} \frac{\partial \tau}{\partial s}+\left(c \dot{d}_{y}+d_{y} \dot{s} \frac{\partial c}{\partial s}\right) \frac{T \theta_{i e}}{C \theta_{d e}} \\
& -\left(\dot{\theta}_{i e}\left(C \theta_{i e}\right)^{-1}+\dot{\theta}_{d e} T \theta_{d e} S \theta_{i e}\right) v \dot{s}^{-1}
\end{aligned}
$$

where $\theta_{d e}$ and $\theta_{i e}$. must differ from $\frac{\pi}{2}[\pi]$ and $d_{y}$ from $\frac{1}{c}$.

Replacing $\dot{s}, \dot{d}_{y}, \dot{\theta}_{i e}$, and $\dot{\theta}_{d e}$ by their values in (4), the input $u_{3}$ is obtained in function of the steering angular velocities $\Omega_{z}$ and $\Omega_{y}$ :

$$
u_{3}=\gamma_{31} \Omega_{y}+\gamma_{32} \Omega_{z}+\gamma_{33}
$$

where $\gamma_{31}, \gamma_{32}$, and $\gamma_{33}$ are defined as 


$$
\begin{aligned}
\gamma_{31}= & -v \dot{s}^{-1} C \delta_{\theta_{d}}\left(C \theta_{i e}\right)^{-1} \\
\gamma_{32}= & v \dot{s}^{-1} C\left(\theta_{i e}\right)^{-1}\left(S \delta_{\theta_{i}} S \delta_{\theta_{d}}-C \delta_{\theta_{i}} S \theta_{i e} T \theta_{d e}\right) \\
\gamma_{33}= & \left(-d_{y} \frac{\partial \tau}{\partial s}-\left(d_{z} \tau+2\left(1-c d_{y}\right) T \theta_{d e}\right)\right. \\
& \left.\left(\tau+c \frac{T \theta_{i e}}{C \theta_{d e}}\right)+d_{y} \frac{\partial c}{\partial s} \frac{T \theta_{i e}}{C \theta_{d e}}\right) \dot{s}+\left(1-c d_{y}\right) \\
& \left(\dot{\delta}_{\theta_{i}} C \delta_{\theta_{d}}-\dot{\delta}_{\theta_{d}} S \theta_{i e} T \theta_{d e}\right)
\end{aligned}
$$

This concludes the proof of Lemma 4.1.

Note that $\gamma_{33}$ depends on the total linear velocity $v$ and also on the derivatives of the compensation inclination and direction angles $\dot{\delta}_{\theta_{d}}$ and $\dot{\delta}_{\theta_{i}}$, respectively, which represent the interaction with the environment. To compute these parameters, several control strategies can be used such as a derivation of an observer for drift compensation as in Lenain et al. (2006) or introduction of the dynamics in the controller as in Lenain et al. (2010). As the experimental environment in this paper is stable, $\delta_{\theta_{i}}$ and $\delta_{\theta_{d}}$ can be considered as constants and the minor disturbances arising from this choice can be compensated using the vision feedback.

Lemma 4.2. Introducing a change in time-scale defined by

$$
\forall i=1, \cdot 5, \frac{\dot{z}_{i}}{\left|\dot{z}_{1}\right|}=z_{i}^{\prime}
$$

and defining the following time-independent control inputs:

$$
\begin{aligned}
& u_{12}=\frac{u_{2}}{u_{1}} \\
& u_{13}=\frac{u_{3}}{u_{1}}
\end{aligned}
$$

the chained form system (11) can be converted into the following linear time-invariant (LTI) model:

$$
\begin{aligned}
z_{1}^{\prime} & =\operatorname{sign}\left(u_{1}\right) \\
z_{2}^{\prime} & =\operatorname{sign}\left(u_{1}\right) z_{3} \\
z_{3}^{\prime} & =\operatorname{sign}\left(u_{1}\right) u_{12} \\
z_{4}^{\prime} & =\operatorname{sign}\left(u_{1}\right) z_{5} \\
z_{5}^{\prime} & =\operatorname{sign}\left(u_{1}\right) u_{13}
\end{aligned}
$$

These changes are defined for $u_{1}$ constant and non-zero.

Proof. Applying (21) and (22) to (11) trivially yields (23), which can be rewritten as follows:

$$
\begin{aligned}
{\left[\begin{array}{c}
z_{2}^{\prime} \\
z_{3}^{\prime} \\
z_{4}^{\prime} \\
z_{5}^{\prime}
\end{array}\right]=} & {\left[\begin{array}{cccc}
0 & \operatorname{sign}\left(u_{1}\right) & 0 & 0 \\
0 & 0 & 0 & 0 \\
0 & 0 & 0 & \operatorname{sign}\left(u_{1}\right) \\
0 & 0 & 0 & 0
\end{array}\right]\left[\begin{array}{l}
z_{2} \\
z_{3} \\
z_{4} \\
z_{5}
\end{array}\right] } \\
+ & {\left[\begin{array}{cc}
0 & 0 \\
\operatorname{sign}\left(u_{1}\right) & 0 \\
0 & 0 \\
0 & \operatorname{sign}\left(u_{1}\right)
\end{array}\right]\left[\begin{array}{l}
u_{12} \\
u_{13}
\end{array}\right] }
\end{aligned}
$$

The latter is, thus, trivially LTI.

\subsection{Control}

Proposition 4.1. Under the conditions set out in Lemmas 4.1 and 4.2, the control

$$
\begin{gathered}
{\left[\begin{array}{l}
u_{12} \\
u_{13}
\end{array}\right]=-\left[\begin{array}{cc}
k_{d 1} & 0 \\
0 & k_{d 2}
\end{array}\right]\left[\begin{array}{l}
z_{2} \\
z_{4}
\end{array}\right]} \\
-\left[\begin{array}{cc}
\operatorname{sign}\left(u_{1}\right) k_{t 1} & 0 \\
0 & \operatorname{sign}\left(u_{1}\right) k_{t 2}
\end{array}\right]\left[\begin{array}{l}
z_{3} \\
z_{5}
\end{array}\right]
\end{gathered}
$$

where $k_{t 1}, k_{d 1}, k_{t 2}$, and $k_{d 2}$ are the control gains and are strictly positive, providing second-order time-independent closed-loop dynamics to stabilize the system (24).

Proof. Replacing the control law (25) in the linear system (23) gives the following equation:

$$
z_{3}^{\prime}=-\operatorname{sign}\left(u_{1}\right) k_{d 1} z_{2}-k_{t 1} z_{3}
$$

From $(23), z_{3}=\operatorname{sign}\left(u_{1}\right) z_{2}^{\prime}$ and because $u_{1}$ is constant and non-zero, $z_{3}^{\prime}=\operatorname{sign}\left(u_{1}\right) z_{2}^{\prime \prime}$.

Inserting those in the above yields

$$
\operatorname{sign}\left(u_{1}\right)\left[z_{2}^{\prime \prime}+k_{t 1} z_{2}^{\prime}+k_{d 1} z_{2}\right]=0
$$

Similar derivation applies to $z_{5}$ and $z_{4}$ and, therefore, the linear system (23) is equivalent to the following two decoupled one-dimensional second-order systems:

$$
\begin{aligned}
& z_{2}^{\prime \prime}+k_{t 1} z_{2}^{\prime}+k_{d 1} z_{2}=0 \\
& z_{4}^{\prime \prime}+k_{t 2} z_{4}^{\prime}+k_{d 2} z_{4}=0
\end{aligned}
$$

This concludes the proof of Proposition 4.1.

Reverting the changes of variables in Lemma 4.1 allows us to compute the actual inputs from the actual outputs:

$$
\begin{aligned}
& v(t)=\text { arbitrary } \neq 0 \\
& \left(\Omega_{y}, \Omega_{z}\right)=f\left(d_{y}, d_{z}, \theta_{i e}, \theta_{d e}\right)
\end{aligned}
$$

with $f\left(d_{y}, d_{z}, \theta_{i e}, \theta_{d e}\right)$ expressed in the following section.

\subsection{Actuation}

The stabilizing time-independent control inputs $u_{12}$ and $u_{13}$ given by (25) allow us to compute

$$
\begin{aligned}
u_{2} & =u_{12} u_{1} \\
& =-k_{d 1} u_{1} z_{2}-k_{t 1}\left|u_{1}\right| z_{3} \\
& =-k_{d 1} u_{1} d_{y}-k_{t 1}\left|u_{1}\right|\left(\tau d_{z}+\left(1-c d_{y}\right) T \theta_{d e}\right)
\end{aligned}
$$

and

$$
\begin{aligned}
u_{3} & =u_{13} u_{1} \\
& =-k_{d 2} u_{1} z_{4}-k_{t 2}\left|u_{1}\right| z_{5} \\
& =-k_{d 2} u_{1} d_{z}-k_{t 2}\left|u_{1}\right|\left(-\tau d_{y}+\left(c d_{y}-1\right) C\left(\theta_{d e}\right)^{-1} T \theta_{i e}\right)
\end{aligned}
$$


Recall that from (16) and (20), the latter have the following expressions:

$$
\begin{aligned}
& u_{2}=\gamma_{21} \Omega_{z}+\gamma_{22} \\
& u_{3}=\gamma_{31} \Omega_{y}+\gamma_{32} \Omega_{z}+\gamma_{33}
\end{aligned}
$$

By knowing the position of the helical swimmer ${ }^{u} \mathbf{p}_{G}$ and the path parameters $(s, \tau$, and $c)$, the steering angular velocities $\Omega_{y}$ and $\Omega_{z}$ can be computed by inverting (28)

$$
\begin{aligned}
& \Omega_{z}=\left(u_{2}-\gamma_{22}\right) \gamma_{21}^{-1} \\
& \Omega_{y}=\left(u_{3}-\gamma_{33}-\gamma_{32} \gamma_{21}^{-1}\left(u_{2}-\gamma_{22}\right)\right) \gamma_{31}^{-1}
\end{aligned}
$$

Asymptotic stability of system (23) under control (25) is achieved by simply tuning the coefficients of two decoupled single-input single-output (SISO) second-order systems (Proposition 4.1). From Lemma 4.2, this ensures the stability of the chained form system (11) under $u_{2}$ (26) and $u_{3}$ (27), trivially obtained from (22) and (25). Stabilizing (11) stabilizes (9) thanks to their equivalence (Lemma 4.1) and, thus, spatial path following can be achieved.

\subsection{Self-rotation and steering controls of the helical swimmer}

The helical swimmer is driven by a rotating magnetic field B generated thanks to the electromagnetic system presented in Figure 1, which can be decomposed as follows:

$$
\mathbf{B}=\mathbf{B}_{\|}+\mathbf{B}_{\perp}
$$

where $\mathbf{B}_{\perp}$ and $\mathbf{B}_{\|}$are the magnetic field parallel and perpendicular to the swimming axis, respectively.

To achieve a rotation about the helical robot principal axis $\mathbf{x}_{B}$, the required torque must satisfy (5). Thereby, both the magnetization $\mathbf{M}$ and the magnetic field $\mathbf{B}_{\perp}$ must lie in a plane perpendicular to the swimming axis $\Omega_{x} \mathbf{C}^{-1} \mathbf{x}_{B}$. The identification of $\mathbf{C}$ is difficult because of manufacturing errors and environmental disturbances. For that, $\mathbf{C}$ is chosen as an identity matrix and the errors arising from this choice can be compensated for by the experimental set-up vision feedback. The swimming axis is, thus, approximated to $\Omega_{x} \mathbf{x}_{B}$ along the helical swimmer axis. This approximation has proved its effectiveness in several works such as Morozov and Leshansky (2014) and Xu et al. (2015).

Furthermore, the magnetic field $\mathbf{B}_{\perp}$, which yields the open-loop self-rotation of the helical robot, can be expressed as follows:

$$
\mathbf{B}_{\perp}=B_{0} \cos (2 \pi f t) \mathbf{y}_{B}+B_{0} \sin (2 \pi f t) \mathbf{z}_{B}
$$

where $f$ is the rotation frequency, $B_{0}$ is the magnetic flux density in the center of the workspace, whereas $\mathbf{y}_{B}$ and $\mathbf{z}_{B}$ are the basis vectors of the plane perpendicular to the helical swimmer axis $\mathbf{x}_{B}$.

In $\mathrm{Xu}$ et al. (2015), the magnetic field $\mathbf{B}_{\|}$, which leads to the steering of the helical robot, is defined as a controller proportional to the geodesic error $\left\|\mathbf{x}_{B} \times \mathbf{x}_{B}^{*}\right\|$ and is given as

$$
\mathbf{B}_{\|}=-\operatorname{sign}\left(\mathbf{B}_{\perp} \mathbf{x}_{B}^{*}\right) \lambda\left\|\mathbf{x}_{B} \times \mathbf{x}_{B}^{*}\right\| \mathbf{x}_{B}
$$

where $\lambda$ is the control gain and $\mathbf{x}_{B}^{*}$ is the desired orientation of the helical robot, which is reconstructed thanks to the time integration of the steering angular velocity $\boldsymbol{\Omega}$.

In this work, we use a more economical approach that consists of incorporating directly the steering angular velocities in the magnetic field $\mathbf{B}_{\|}$without going through the time integration of the steering angular velocities. Thus, the angular error can be rewritten as

$$
\begin{aligned}
\mathbf{x}_{B} & \times \mathbf{x}_{B}^{*}=\mathbf{x}_{B} \times\left(\mathbf{x}_{B}+\left(\boldsymbol{\Omega} \times \mathbf{x}_{B}\right) d t\right) \\
& =\mathbf{x}_{B} \times\left(\boldsymbol{\Omega} \times \mathbf{x}_{B}\right) d t \\
& =\left(\mathbf{I}-\mathbf{x}_{B} \mathbf{x}_{B}^{\mathrm{T}}\right) \boldsymbol{\Omega} d t
\end{aligned}
$$

where $\mathbf{I}$ is a $3 \times 3$ identity matrix and $d t$ the sample time. Finally, the magnetic field $\mathbf{B}_{\|}$, which is thus the actuator associated to the steering angular velocity $\boldsymbol{\Omega}$ in (29), can be expressed as

$$
\mathbf{B}_{\|}=-\operatorname{sign}\left(\left(\mathbf{B}_{\perp} \times \boldsymbol{\Omega}\right) \mathbf{x}_{B}\right) \lambda\left\|\left(\mathbf{I}-\mathbf{x}_{B} \mathbf{x}_{B}^{\mathrm{T}}\right) \boldsymbol{\Omega}\right\| \mathbf{x}_{B}
$$

The helical swimmer pose (i.e. ${ }^{u} \mathbf{p}_{G}, \theta_{d}$, and $\theta_{i}$ ) is reconstructed by vision. In fact, the components of the helical swimmer axis $\mathbf{x}_{B}$ can be computed using the two angles $\theta_{d}$ and $\theta_{i}$ as shown in Figure 2 by

$$
\mathbf{x}_{B}=\left[\begin{array}{lll}
C \theta_{i} C \theta_{d} & C \theta_{i} S \theta_{d} & S \theta_{i}
\end{array}\right]
$$

\section{Experimental results}

The 3D visual control developed above has been implemented on the prototype in different paths with different complexities to evaluate the effectiveness of the controller and the helical swimmer to follow these kinds of trajectories.

To control the 3D helical swimmer displacements in closed-loop, the procedure presented in Algorithm 1 has been developed.

\section{1. $3 D$ trajectories}

The 3D visual servo control is tested in different curve shapes, starting by a 3D straight line without any curvature or torsion as shown in Figure 6(a). The helical swimmer was initially out of the reference path and it can be seen that the helical swimmer converges and straightens gradually to reach the desired path, then moves along the path. By comparison with the straight line following realized in $\mathrm{Xu}$ et al. (2015) where the swimmer regulates the lateral errors to zero whereas the altitude is controlled in open-loop, the 3D controller proposed in this paper servoed both the altitude and lateral errors to zero. Furthermore, the controller was tested with more complex trajectories such as a helix with a constant curvature and torsion and an inclined sinusoidal 


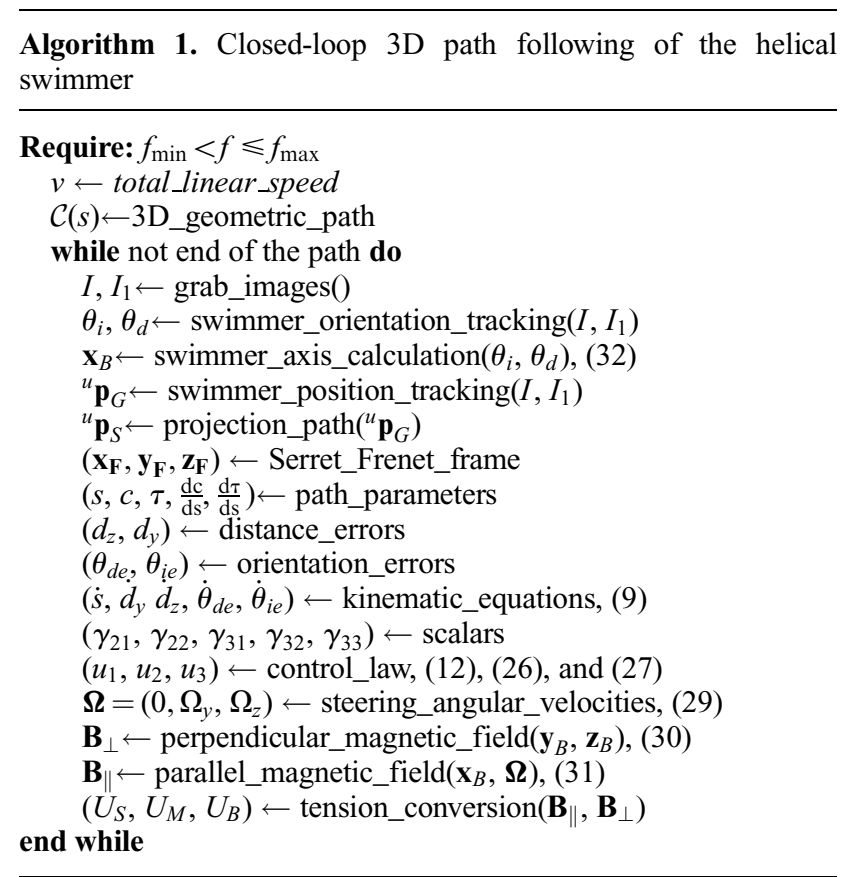

trajectory with variable curvature and inclination as shown in Figure 6(b) and (c), respectively (see also Extension 1). It can be shown that the controller is still efficient despite the complexity and succession of curvatures. More details about these trajectories are reported in Oulmas et al. (2016a).

\subsection{Decoupling path following and velocity profile}

For most applications, the tasks to be performed by helical microswimmers will be defined as a geometric path to follow without specification of the velocity profile. These two tasks together (geometric path and velocity profile) define a trajectory tracking. However, in trajectory tracking, disturbances result in time delay, and to overcome this, the swimmer cuts the trajectory to reach the desired position with the desired time. Thus, the geometrical path will be not achieved (reduced accuracy).
During disturbances, decoupling these two tasks will yield better performances with regard to accuracy. In addition, the swimmer velocity can be modified independently of the path as a function of the task and the environment (through the Reynolds number).

In this subsection, experiments have been performed using an inclined sinusoidal trajectory with different velocity profiles to illustrate the impact of the velocity on the path following of the helical swimmer.

Therefore, the total linear velocity $v$ depends on the rotation frequency $f$ and the inclination angle $\theta_{i}$ of the helical swimmer. However, the angle $\theta_{i}$ is already used as a control input. Therefore, only the rotation frequency can be used to change the swimmer velocity $v$.

To do so, two different frequency profiles are generated. The first is a rectangular signal (Figure 7a) and the second is a continuous arbitrary signal (Figure $7 b$ ).

The results are compared with the same path using a constant frequency profile, and are reported in Table 2. It shows that the performances of the path following are still efficient in all three cases. As a conclusion, whereas the rotation frequency is between $f_{\min }$, the minimum frequency required to compensate for the weight of the helical robot in the fluid, and $f_{\text {cut off }}$, the cut-off frequency (Xu et al., 2014), the variation of the velocity profile has no effects on the performance of the path following.

\subsection{Impact of the viscosity of the fluid on the path following}

The helical swimmer dynamics depend severely on its geometry and the viscosity of the fluid. This is represented in the coefficients of the mobility matrix (3) (see Mahoney et al. (2011) for more details). The fluid viscosity is affected by the temperature. Therefore, the closed-loop control presents a good solution to compensate for disturbances caused by the change in viscosity and thermal noises.

To obtain the same conditions as at low scale in terms of Reynolds numbers, the helical swimmer was emerged in glycerol, which is a viscous liquid. Furthermore, experiments using an inclined sinusoidal trajectory with different liquid viscosities have been performed to illustrate the

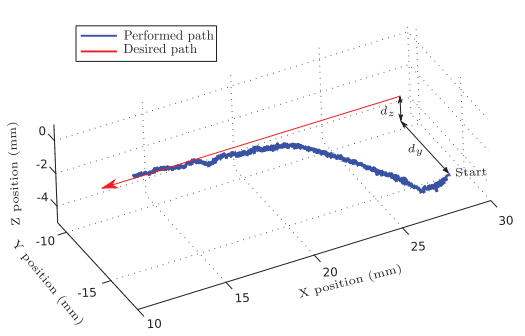

(a)

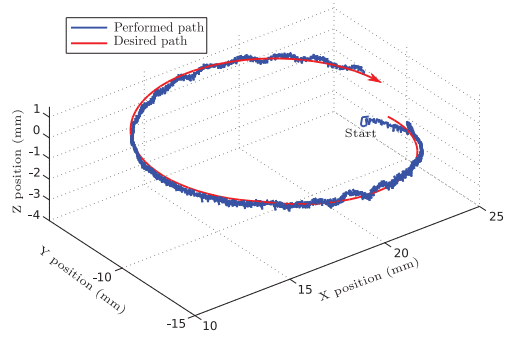

(b)

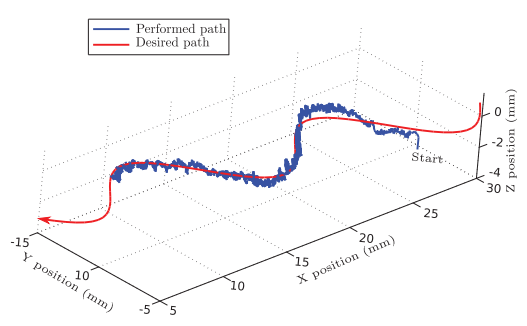

(c)

Fig. 6. Path following of different trajectories in space using a scaled-up helical microswimmer: (a) 3D straight line; (b) helix trajectory; (c) inclined sinusoidal trajectory. 


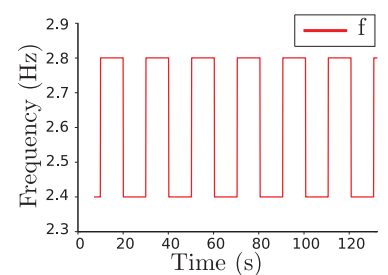

(a)

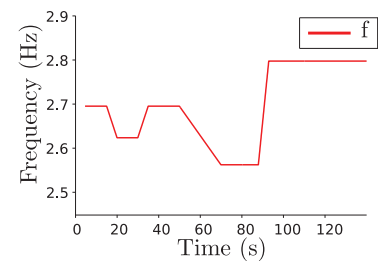

(b)
Fig. 7. Velocity variation of the helical swimmer using different frequency profiles such as (a) a rectangular signal and (b) a continuous arbitrary signal.

effectiveness of the closed-loop control to the change in viscosity.

During the experiments, the compensation inclination angle and the control gains are kept fixed. However, to balance the weight of the helical robot, the swimmer vertical velocity (i.e. the rotation frequency) is increased in function of the glycerol viscosity. Table 2 summarize the frequencies used for different glycerol viscosities.

The results of following an inclined sinusoidal trajectory with three different viscosities are compared using the mean and standard deviation of the distance and orientation errors as shown in Figure 8.

The graph shows that the path following is accurate in all three cases with an error of less than $0.5 \mathrm{~mm}$. As the path following and the velocity profile are decoupled, the strong disturbances caused by the change in the fluid viscosity can be corrected by increasing or decreasing the helical swimmer speed, hence, the interest of decoupling "path following" and "velocity profile."

In the next subsection, experiments demonstrating the effect of lateral disturbances on the performance of the path following are performed.

\subsection{Boundary effects}

At low scale, microswimmers are affected by several disturbances such as boundary effects. Indeed, many experiments have shown that close to a solid surface, microswimmers are attracted or repulsed by these boundaries.

In this subsection, we tested the effectiveness of the 3D controller to these disturbances by analyzing the effect of a solid plane wall. A reference straight line is generated with a distance $\delta$ to a solid surface. The helical swimmer swims parallel to the wall, as illustrated in Figure 9.

It can be noticed that the controller is efficient for a path-wall distance over $2.5 \mathrm{~mm}$. Under this distance, the accuracy decreases dramatically because the helical swimmer hits the wall and rolls on the surface when trying to reach the reference path. But, this does not call into question the controller robustness.

Other experiments putting the helical swimmer in contact with the solid surface were performed in order to show that the controller is robust to boundaries.
Table 2. The distance $(\mu \mathrm{m})$ and orientation (rad) errors during the inclined sinusoidal trajectory following with different velocity profiles.

\begin{tabular}{llllll}
\hline Velocity & Errors & $d_{y}$ & $d_{z}$ & $\theta_{d e}$ & $\theta_{i e}$ \\
\hline constant & RMS & 178 & 396 & 0.597 & 0.079 \\
& SD & 173 & 337 & 0.594 & 0.068 \\
rectangular & RMS & 292 & 301 & 0.255 & 0.108 \\
signal & SD & 281 & 242 & 0.254 & 0.099 \\
arbitrary & RMS & 211 & 330 & 0.308 & 0.153 \\
signal & SD & 182 & 277 & 0.305 & 0.106 \\
\hline
\end{tabular}

Table 3. The rotation frequency of the helical swimmer for different viscosities of the fluid at $20^{\circ} \mathrm{C}$.

\begin{tabular}{lll}
\hline Glycerol & Viscosity (mPa.s) & Frequency $(\mathrm{Hz})$ \\
\hline $99.99 \%$ & 1,412 & 2.3 \\
$99 \%$ & 1,150 & 2.5 \\
$98 \%$ & 939 & 3 \\
\hline
\end{tabular}

In the first experiment, the helical swimmer is initially in contact entirely with the bottom surface of the beaker as shown in Figure 10a (Extension 2). During the path following, it can be seen that the robot manages to detach itself from the solid surface and then converges to the reference path as illustrated in Figure 10b (Extension 2). The contact with the bottom surface makes the robot roll a little on the surface in lateral displacements. However, despite the disturbance, the controller is still efficient.

In the second experiment, the robot is placed entirely in contact with the side surface of the beaker as shown in Figure 11. It can be seen that when the robot is on the solid surface, it rolls upwards until it detaches and then converges to the reference straight line. Finally, the experiments show that the controller is robust to boundary effects.

\subsection{Open-loop versus closed-loop}

As shown previously, the 3D control performance of the helical swimmer depends on the viscosity of the liquid and the boundary effects caused by the beaker wall. Comparing the closed-loop control and the open-loop control allows us to illustrate the different disturbances that the helical robot undergoes during the path following.

The reference path is a straight line with a constant altitude. The helical swimmer is initially on the path. As shown in Figure 12, using the open-loop algorithm, the helical swimmer drifts from the desired path because of: the imperfection of the system such as magnetic field gradient; modeling errors, namely the propulsion matrix and compensation inclination angle; the friction between the robot and the substrate; boundary effects; variation of the temperature. 


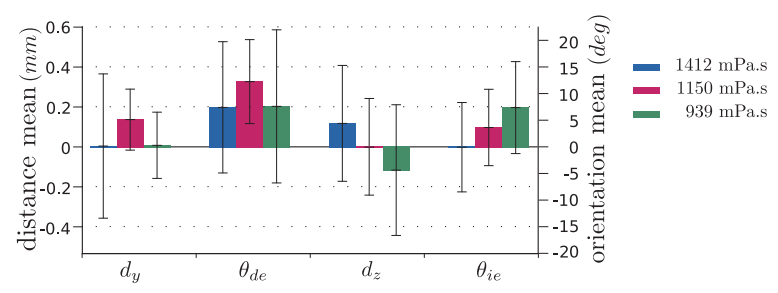

Fig. 8. The mean and standard deviation of the distance and orientation errors while following an inclined sinusoidal trajectory for three different viscosities. The tests were carried out several times.

On the other hand, using the closed-loop control, the helical swimmer shows better performance despite disturbances, with an accuracy less than $0.2 \mathrm{~mm}$ (see Extension 3). In lab-on-chips and biomedical tasks, the motion of the helical microswimmer should be robust and accurate and using the closed-loop control allows the environmental disturbances such as high flows in arteries to be overcome or obstacles to be avoided.

\subsection{The helical swimmer weight compensation}

To compensate for the weight of the helical robot, it is inclined by some angles with respect to the horizontal plane. This is referenced by the so-called compensation inclination angle $\delta_{\theta_{i}}$, which is defined as the angle between the principal axis and the total linear velocity $\mathbf{v}$ of the helical robot. Below this angle, the helical robot loses altitude and gains speed while descending owing to gravity. Over this angle, the helical robot gains altitude and loses some speed, as illustrated in Figures 13 and 14.

In Figure 13, the helical swimmer follows a sinusoidal trajectory in the vertical plane (Extension 4) and it can be seen that when the inclination angle is greater than the compensation inclination angle, the robot increases in altitude and when the inclination angle is lower than the compensation inclination angle the robot decreases in altitude.

The compensation inclination angle $\delta_{\theta_{i}}$ is controlled in open-loop and can be estimated by vision by computing the vertical and horizontal velocities $v_{\text {ver }}$ and $v_{\text {hor }}$, respectively, as follows:

$$
\delta_{\theta_{i}}=\tan \left(\frac{v_{\mathrm{ver}}}{v_{\text {hor }}}\right)^{-1}
$$
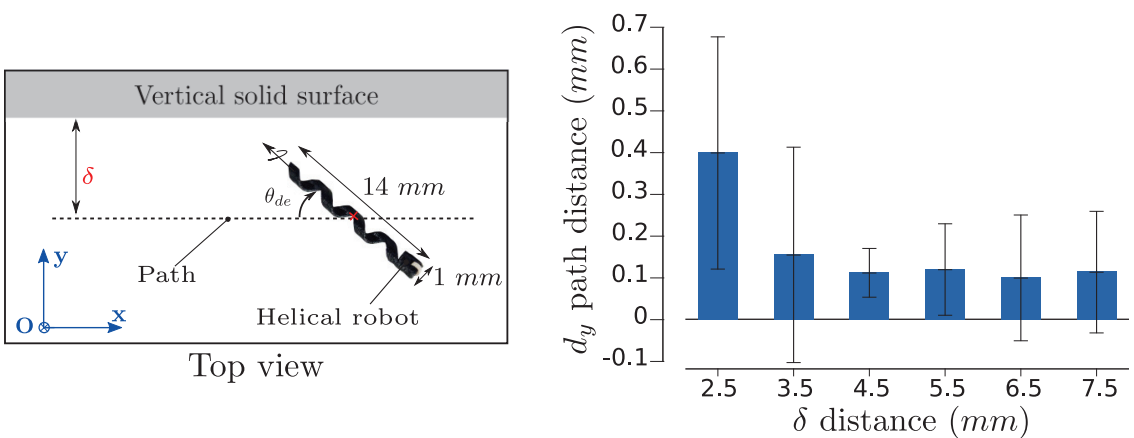

Fig. 9. Effect of a vertical solid surface on the performance of the path following of the helical swimmer. At each wall distance $\delta$, the mean and standard deviation errors of the lateral distance $d_{y}$ are shown.

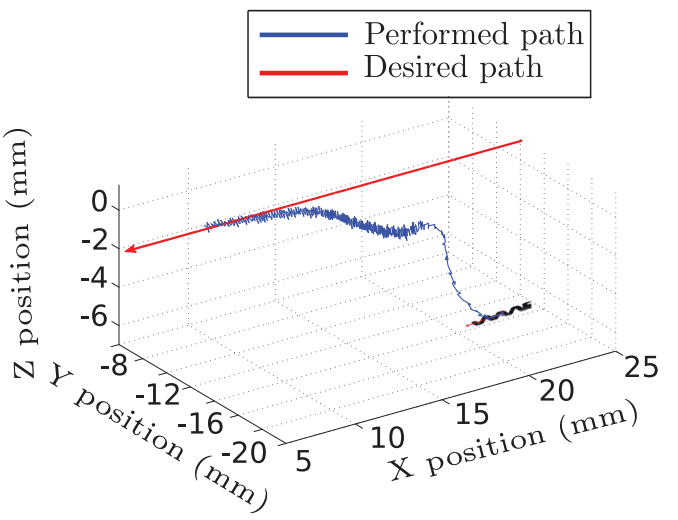

(a)



(b)

Fig. 10. Experiment 1: The interaction between the helical swimmer and the bottom surface of the beaker (a) and the distance errors during the path following (b). 


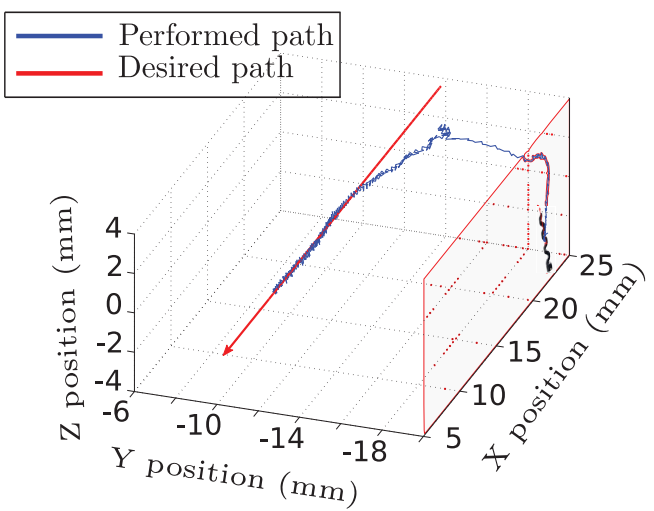

(a)

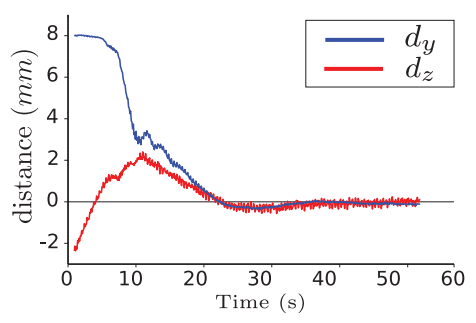

(b)

Fig. 11. Experiment 2: The interaction between the helical swimmer and the side surface of the beaker (a) and the distance errors during the path following (b).

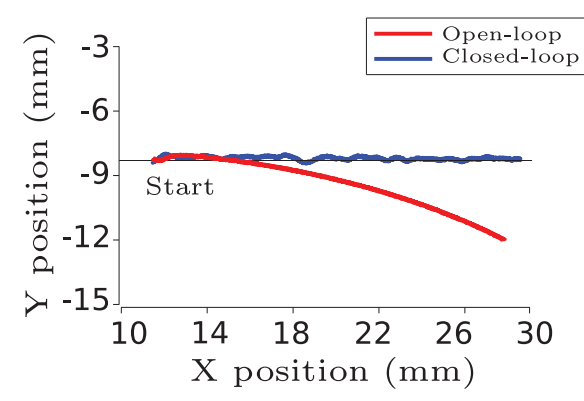

(a)

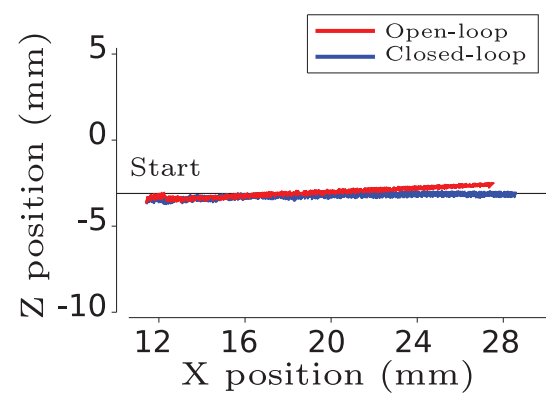

(b)

Fig. 12. Comparison between the open-loop control and the visual servo control: (a) horizontal plane; (b) vertical plane.

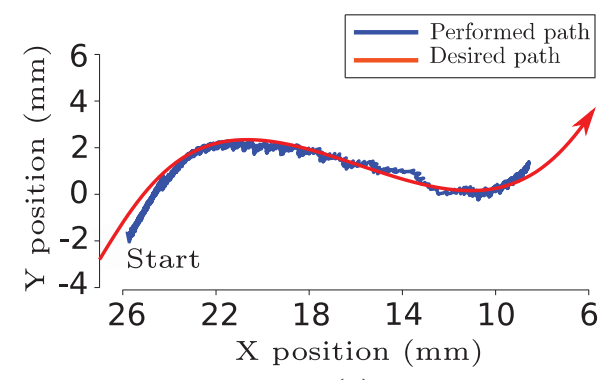

(a)

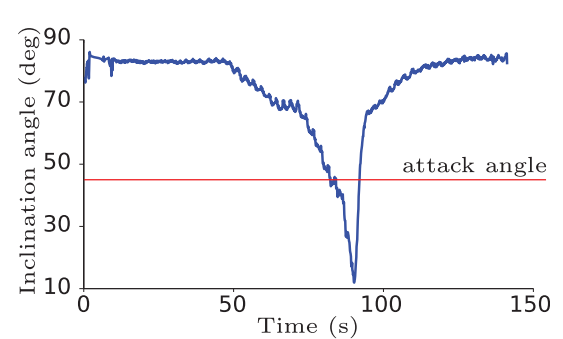

(b)

Fig. 13. Sinusoidal trajectory following in the vertical plane using a scaled-up helical microswimmer with a compensation inclination angle of $45^{\circ}$ (a) and the evolution of the inclination angle of the helical swimmer (b).

The computing errors of the compensation inclination angle will be corrected thanks to the closed-loop control.

To show the effects of this angle on the performance of the path following, a series of test with different compensation inclination angles was performed using the sinusoidal trajectory with constant altitude, while maintaining the same rotation frequency and control gains during the experiments (Figure 15).
In the horizontal plane (Figure 15a), all trajectories converge to the desired path, the compensation inclination angle has no specific effects on the performance of the path following on the horizontal plane. However, in the vertical plane (Figure 15b), all trajectories converge to the reference path with a more or less error depending on the compensation inclination angle. The mean and standard deviation of the distance and orientation errors 


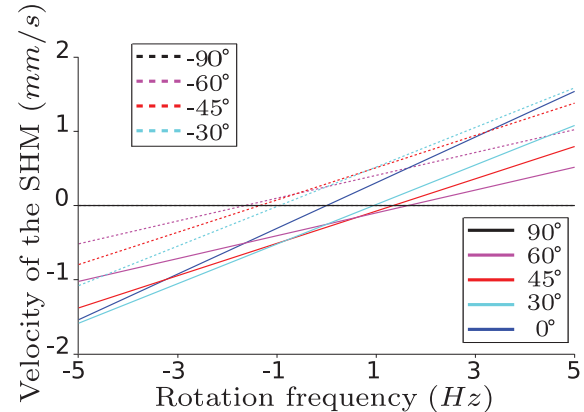

(a)



(b)

Fig. 14. Estimated linear velocities of the helical robot with different inclination angles in pure glycerol: (a) horizontal plane; (b) vertical plane.

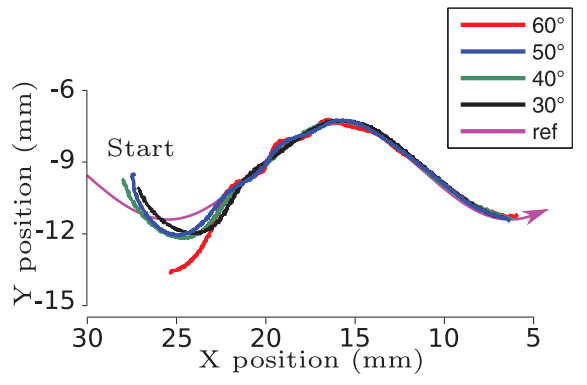

(a)

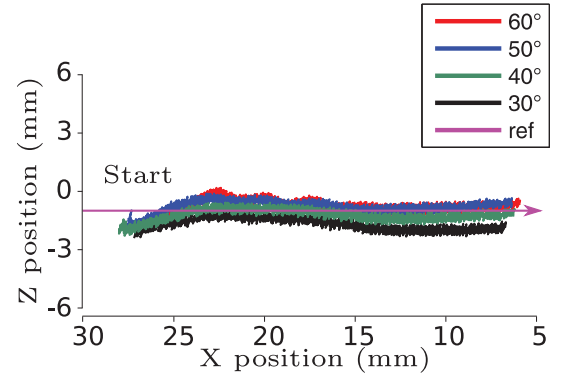

(b)

Fig. 15. Trajectories of the helical swimmer using the $3 \mathrm{D}$ closed-loop control with different compensation inclination angles: (a) horizontal plane; (b) vertical plane.

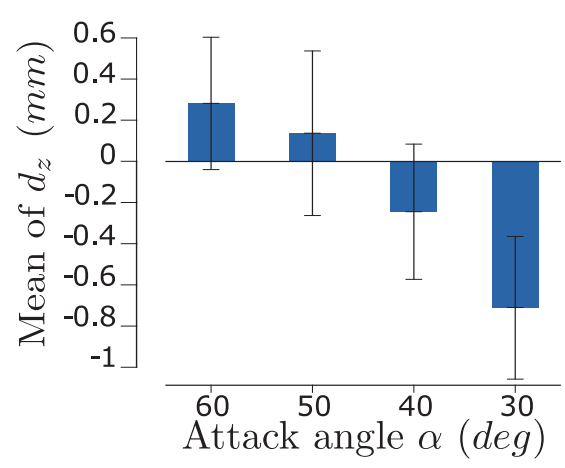

(a)

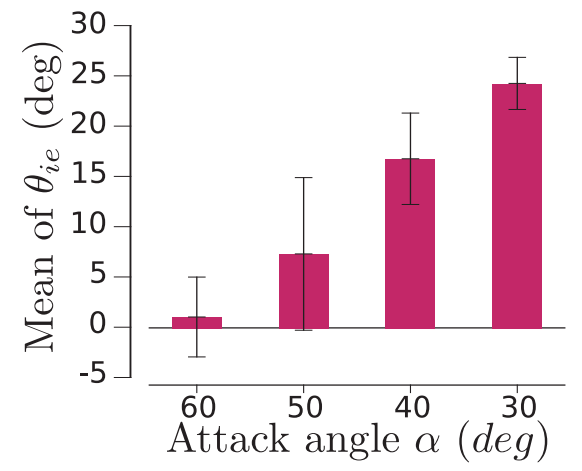

(b)

Fig. 16. Mean and standard deviation of the (a) distance and (b) orientation errors in the vertical plane, as a function of the compensation inclination angle.

in the vertical plane are depicted in Figure 16 to compare the different paths.

The experimental results show that the accuracy is quite satisfactory for a compensating angle between $40^{\circ}$ and $60^{\circ}$, as illustrated in Figure 16a. Indeed, the closed-loop control tends to correct the compensation inclination angle computing errors by increasing the inclination angle as shown in Figure 16b. Finally, the closed-loop control can be used to compensate the computing errors of the artificial helical swimmer weight and uncertainties in general.

\subsection{Gain tuning}

The performance of the closed-loop controller (stability, accuracy, and rapidity) can be adjusted using the control gains $k_{d 1,2}$ and $k_{t 1,2}$. Several experiments using the same 


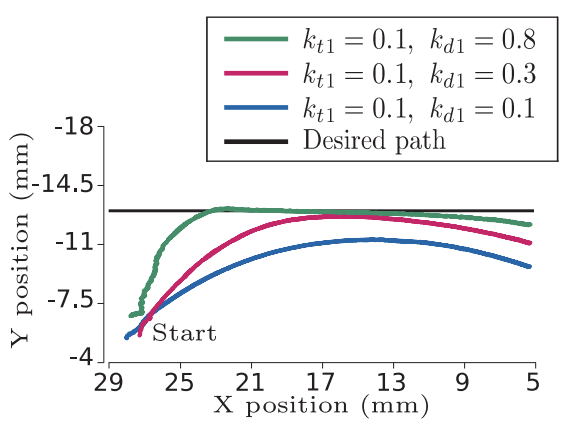

(a)



(b)

Fig. 17. The projection of the tracked helical swimmer path while following an inclined sinusoidal trajectory on (a) the horizontal plane and (b) the vertical plane.

path with different control gains are performed to illustrate the effect of these parameters on the performance of the path following. Trajectories of the helical robot while following straight lines with different control gains in the horizontal and vertical planes are depicted in Figure 17.

The helical swimmer is initially off the path. It can be noticed that the helical swimmer converges to the path more or less rapidly depending on the parameters $k_{d 1}$ and $k_{d 2}$. In addition, the error due to the lateral disturbances at the end of the trajectory in Figure 17a decreases by increasing the gain $k_{d 1}$. On the other side, the gains $k_{t 1}$ and $k_{t 2}$ play a stabilizing role. In fact, the parameters $k_{d 1,2}$ and $k_{t 1,2}$ can be considered as the proportional and derivative gains of a PD controller.

\section{Conclusion}

3D path following of magnetic helical swimmers is realized for the first time in closed-loop. The robot is modeled in a local frame using the Serret-Frenet frame, taking into account the weight of the robot and lateral disturbances. The decoupled stable controller is designed using a new chained formulation to linearize the kinematic model.

This approach is validated and analyzed using a scaledup helical swimmer actuated magnetically. Several conditions are tested: different velocity profiles, complex curves, viscosities, gains, boundary effects, and compensation inclination angles.

The experiments have shown that the path-following controller is robust and accurate against disturbances such as boundary effects that are significant at low Reynolds numbers. In lab-on-chips, it can be used to transport objects to a desired location with accuracy while avoiding obstacles. For more complex situations with dynamic obstacles, a motion planning technique, which is a combination of path planning with smooth constraints and path following control, could be a robust solution to avoid collisions with obstacles.

In the future, we must find a robust identification method of the mobility matrix especially the submatrix $\mathbf{C}$ and make a comparison to see the effect of neglecting the submatrix $\mathbf{C}$ in the magnetic controller $\mathbf{B}$ on the helical robot performance. In addition, controlling a group of multiple magnetic microswimmers is interesting to improve their performances such as targeted drug delivery inside the human body using several microrobots.

\section{Acknowledgment}

The authors would like to warmly thank Pascal Morin for his valuable help in proof checking the derivation of chained form.

\section{Funding}

This work was supported by Région Franche-Comté and the French National Research Agency, through the EIPHI Graduate School (contract "ANR-17-EURE-0002") and ANR MultiFlag (grant number ANR-16-CE33-0019).

\section{References}

Aguiar A and Hespanha J (2007) Trajectory-tracking and pathfollowing of underactuated autonomous vehicles with parametric modeling uncertainty. IEEE Transactions on Automatic Control 52(8): 1362-1379.

Bushnell L, Tilbury D and Sastry S (1995) Steering three-input chained form nonholonomic systems using sinusoids: The fire truck example. The International Journal of Robotics Research 14(1): 366-381.

Chaillet N and Régnier S (2013) Microrobotics for Micromanipulation. New York: John Wiley \& Sons.

Coey J (2010) Magnetism and Magnetic Materials. Cambridge: Cambridge University Press.

Encarnacao P and Pascoal A (2000) 3D path following for autonomous underwater vehicle. In: IEEE Conference on Decision and Control, Sydney, Australia, pp. 2977-2982.

Etkin B (2012) Dynamics of atmospheric flight. Chelmsford, MA: Courier Corporation.

Fu H, Jabbarzadeh M and Meshkati F (2015) Magnetization directions and geometries of helical microswimmers for linear velocity-frequency response. Physical Review E 91(4): 043011.

Ghosh A and Fischer P (2009) Controlled propulsion of artificial magnetic nanostructured propellers. Nano Letters 9(6): 2243-2245. 
Ghosh A, Paria D, Rangarajan G and Ghosh A (2014) Velocity fluctuations in helical propulsion: How small can a propeller be. Journal of Physical Chemistry Letters 5(1): 62-68.

Healey A and Lienard D (1993) Multivariable sliding-mode control for autonomous diving and steering of unmanned underwater vehicles. IEEE Journal of Oceanic Engineering 18(3): 327-339.

Kaminer I, Yakimenko O, Pascoal A and Ghabcheloo R (2006) Path Generation, path following and coordinated control for time Critical missions of multiple UAVs. In: American Control Conference, Minneapolis, MN, pp. 4906-4913.

Lenain R, Thuilot B, Cariou C and Martinet P (2006) Mobile robot control in presence of sliding: Application to agricultural vehicle path tracking. In: IEEE Conference on Decision and Control, San Diego, CA, pp. 6004-6009.

Lenain R, Thuilot B, Cariou C and Martinet P (2010) Mixed kinematic and dynamic sideslip angle observer for accurate control of fast off-road mobile robots. Journal of Field Robotics 27(2): 181-196.

Mahoney A, Sarrazin J, Bamberg E and Abbott J (2011) Velocity control with gravity compensation for magnetic helical microswimmers. Advanced Robotics 25(8): 1007-1028.

Marchand E, Spindler F and Chaumette F (2005) ViSP for visual servoing: a generic software platform with a wide class of robot control skills. IEEE Robotics and Automation Magazine 12(4): 40-52.

Medina-Sánchez M, Schwarz L, Meyer A, Hebenstreit F and Schmidt O (2015) Cellular cargo delivery: Towards assisted fertilization by spermCarrying micromotors. Nano letters 16(1): 555-561.

Morin P and Samson C (2008) Motion control of wheeled mobile robots. In: Springer Handbook of Robotics. Berlin: Springer, pp. 799-826

Morozov K and Leshansky A (2014) The chiral magnetic nanomotors. Nanoscale 6(3): 1580-1588.

Oliveira T, Encarnacao P and Aguiar A (2013) Moving path following for autonomous robotic vehicles. In: European Control Conference, Zürich, Switzerland, pp. 3320-3325.

Oprea J (2007) Differential Geometry and Its Applications. Washington, DC: Mathematical Association of America.

Oulmas A, Andreff N and Régnier S (2016a) Closed-loop 3D path following of scaled-up helical microswimmers. In: IEEE International Conference on Robotics and Automation, Stockholm, Sweden, pp. 1725-1730.

Oulmas A, Andreff N and Régnier S (2016b) Chained formulation of $3 \mathrm{D}$ path following for nonholonomic autonomous robots in a Serret-Frenet frame. In: American Control Conference, Boston, MA, pp. 7275-7280.

Peyer K, Zhang L, Kratochvil B and Nelson B (2010) Non-ideal swimming of artificial bacterial flagella near a surface. In;
IEEE International Conference on Robotics and Automation, Anchorage, AK, pp. 96-101.

Purcell E (1977) Life at low Reynolds number. American Journal Physics 45(1): 3-11.

Qiu F and Nelson B (2015) Magnetic helical micro-and nanorobots: Toward their biomedical applications. Engineering 1(1): 21-26.

Qiu F, Fujita S, Mhanna R, Zhang L, Simona B and Nelson B (2015) Magnetic helical microswimmers functionalized with lipoplexes for Targeted Gene Delivery. Advanced Functional Materials 25(11): 1666-1671.

Samson C (1995) Control of chained systems application to path following and time-varying point-stabilization of mobile robots. IEEE Transactions on Automatic Control 4(1): 64-77.

Siauve N, Scorretti R, Burais N, Nicolas L and Nicolas A (2003) Electromagnetic fields and human body: a new challenge for the electromagnetic field computation. International Journal for Computation and Mathematics in Electrical and Electronic Engineering 22(3): 457-469.

Tottori S, Zhang L, Qiu F, Krawczyk K, Franco-Obregn A and Nelson B (2012) Magnetic helical micromachines: Fabrication, controlled swimming, and cargo transport. Advanced Materials 24(6): 811-816.

Wadoo S, Sapkota S and Chagachagere K (2012) Optimal control of an autonomous underwater vehicle. In: IEEE International Conference on Systems, Applications and Technology, Farmingdale, NY, pp. 1-6.

Walsh G and Bushnell L (1995) Stabilization of multiple input chained form control systems. Systems and Control Letters 25(3): 227-234.

Xu T, Hwang G, Andreff N and Régnier S (2014) Modeling and swimming property characterizations of scaled-up helical microswimmers. IEEE/ASME Transactions on Mechatronics 19(3): 1069-1079.

Xu T, Hwang G, Andreff N and Régnier S (2014) Characterization of three-dimensional steering for helical swimmers. In: IEEE International Conference on Robotics and Automation, Hong Kong, China, pp. 4686-4691.

Xu T, Hwang G, Andreff N and Régnier S (2015) Planar path following of 3-D steering scaled-Up helical microswimmers. IEEE Transactions on Robotics 31(1): 117-127.

\section{Appendix A. Index to multimedia extensions}

Archives of IJRR multimedia extensions published prior to 2014 can be found at http://www.ijrr.org, after 2014 all videos are available on the IJRR YouTube channel at http:// www.youtube.com/user/ijrrmultimedia

Table of Multimedia Extensions

\begin{tabular}{lll}
\hline Extension & Media type & Description \\
\hline 1 & Video & Following 3D trajectories using a scaled-up helical microswimmer. \\
2 & Video & The impact of the boundaries on the servo control performance. \\
3 & Video & Open-loop control versus closed-loop control. \\
& Video & Compensating for the SHM weight using the compensation inclination angle. \\
\hline
\end{tabular}




\section{Appendix B: The kinematic model computation}

To compute the translation part of the kinematic model, the position ${ }^{u} \mathbf{p}_{G}$ of the helical swimmer in the global frame $\mathcal{F}_{u}$ is used as follows:

$$
{ }^{u} \mathbf{p}_{G}={ }^{u} \mathbf{p}_{S}+{ }^{u} \mathbf{R}_{F}{ }^{F} \mathbf{p}_{G}
$$

Differentiating this equation with respect to time we have

$$
{ }^{u} \mathbf{v}_{d}={ }^{u} \mathbf{v}_{F}+{ }^{u} \mathbf{R}_{F}{ }^{F} \dot{\mathbf{p}}_{G}+{ }^{u} \dot{\mathbf{R}}_{F}{ }^{F} \mathbf{p}_{G}
$$

Expressing this equation in the Serret-Frenet frame we have

$$
{ }^{F} \mathbf{R}_{u}{ }^{u} \mathbf{v}_{d}={ }^{F} \mathbf{v}_{F}+{ }^{F} \dot{\mathbf{p}}_{G}+{ }^{F} \dot{\mathbf{R}}_{F}{ }^{F} \mathbf{p}_{G}
$$

where

$$
\begin{aligned}
& { }^{F} \mathbf{R}_{u}{ }^{u} \mathbf{v}_{d}={ }^{F} \mathbf{R}_{d}{ }^{d} \mathbf{v}_{d} \\
& { }^{F} \dot{\mathbf{R}}_{F}{ }^{F} \mathbf{p}_{G}={ }^{F} \boldsymbol{\omega}_{F} \times{ }^{F} \mathbf{p}_{G}
\end{aligned}
$$

Replacing these parameters gives (7) as follows:

$$
{ }^{F} \mathbf{R}_{d}{ }^{d} \mathbf{v}_{d}={ }^{F} \mathbf{v}_{F}+{ }^{F} \dot{\mathbf{p}}_{G}+{ }^{F} \boldsymbol{\omega}_{F} \times{ }^{F} \mathbf{p}_{G}
$$

Replacing the expressions of these parameters (see Table 1), Equation (7) can be rewritten as follows:

$$
{ }^{F} \mathbf{R}_{d}\left[\begin{array}{c}
v \\
0 \\
0
\end{array}\right]=\left[\begin{array}{c}
\dot{s} \\
0 \\
0
\end{array}\right]+\left[\begin{array}{c}
0 \\
\dot{d}_{y} \\
\dot{d}_{z}
\end{array}\right]+\left[\begin{array}{c}
\tau \dot{s} \\
0 \\
c \dot{s}
\end{array}\right] \times\left[\begin{array}{c}
0 \\
d_{y} \\
d_{z}
\end{array}\right]
$$

Solving these equations for $\dot{s}, \dot{d}_{y}$, and $\dot{d}_{z}$ gives the translation part of the kinematic model (9a), (9b), and (9c).

For the second part concerning the rotation part of the kinematic model, the derivation of rotation matrix ${ }^{F} \mathbf{R}_{d}$ can be used as follows:

$$
{ }^{F} \dot{\mathbf{R}}_{d}={ }^{F} \mathbf{R}_{d} \mathbf{S k}\left({ }^{d} \boldsymbol{\omega}_{d, F}^{r}\right)
$$

The relative angular velocity between $\mathcal{F}_{d}$ and $\mathcal{F}_{F}$ is expressed as follows:

$$
\begin{aligned}
{ }^{d} \boldsymbol{\omega}_{d, F}^{r} & ={ }^{d} \boldsymbol{\omega}_{d}-{ }^{d} \boldsymbol{\omega}_{F} \\
& ={ }^{d} \boldsymbol{\omega}_{B}+{ }^{d} \boldsymbol{\omega}_{d, B}^{r}-{ }^{d} \boldsymbol{\omega}_{F}
\end{aligned}
$$

where

$$
\begin{aligned}
& { }^{d} \boldsymbol{\omega}_{B}={ }^{d} \mathbf{R}_{B} \boldsymbol{\Omega} \\
& { }^{d} \boldsymbol{\omega}_{F}={ }^{d} \mathbf{R}_{F}{ }^{F} \boldsymbol{\omega}_{F} \\
& { }^{d} \boldsymbol{\omega}_{d, B}^{r}=\left[\begin{array}{c}
0 \\
0 \\
\dot{\delta}_{\theta_{d}}
\end{array}\right]+\operatorname{Rot}\left(\mathbf{z},-\delta_{\theta_{d}}\right)\left[\begin{array}{c}
0 \\
-\dot{\delta}_{\theta_{i}} \\
0
\end{array}\right]
\end{aligned}
$$

Computing ${ }^{d} \boldsymbol{\omega}_{d, F}^{r}=\left[\begin{array}{lll}0 & p & q\end{array}\right]^{\mathrm{T}}$, Equation (8) can be rewritten as follows:

$$
\left[\begin{array}{c}
\dot{\theta}_{d e} \\
\dot{\theta}_{i e}
\end{array}\right]=\left[\begin{array}{cc}
C \theta_{i e}^{-1} & 0 \\
0 & 1
\end{array}\right]\left[\begin{array}{l}
p \\
q
\end{array}\right]
$$

Replacing $p$ and $q$ gives the rotation part of the kinematic model (9d) and (9e). 